\title{
Parametric Optimization and Working Fluid Selection of Organic Rankine Cycle (ORC) for Engine Cooling Water Waste Heat Recovery
}

\author{
Chen Bo \\ North University of China, Tai Yuan, China, 030051 \\ China North Engine Research Institute (Tianjin), Tianjin, China, 300400 \\ Wang Genquan, Zhang Limin \\ China North Engine Research Institute (Tianjin), Tianjin, China, 300400
}

\begin{abstract}
In this paper, aimed to utilize engine cooling waste heat completely, thus promoting engine fuel economy significantly, organic Rankine cycle (ORC) system is proposed and investigated in detail. Considering environmental impact, four promising hydrocarbons, namely butane, isobutane, pentane and isopentane are taken into consideration. The results indicate that butane distinguishes among other fluids for the highest value of output power, and its output power is as large as $6.33 \mathrm{~kW}$, besides that its volumetric flow rate is relatively low as well. Pentane and isopentane can acquire a maximum thermal efficiency of $9.5 \%$ or more for the whole operating parameters.
\end{abstract}

KEYWORD: waste heat recovery; engine cooling water; working fluid

\section{INTRODUCTION}

Over decades, the rapid industrial and economic development has resulted in increasingly needs for energy supply. In the meanwhile, energy shortage and environment pollution are becoming more and more serious. As the main fuel consumption subject, internal combustion engine (ICE) consumes approximately $60-70 \%$ of the total fossil fuel in China. Hence, improving fuel utilization efficiency is regarded as a crucial strategy to meet these challenges. Generally, the efficiency of ICE is difficult to be $42 \%$ or more and according to energy balance research results, around 2/3 of fuel energy is lost in the form of waste heat through exhaust gases and cooling water. Based on experimental investigations for a naturally aspirated gasoline engine, theoretically, the maximum overall fuel efficiency can come up to $60 \%$ through waste heat recovery (WHR) [1]. Thus, WHR is widely accepted as a promising field and numerous scholars show their interests towards it [2-8].

Organic Rankine cycle (ORC) has been proposed as a desirable approach to convert low-medium temperature waste heat, such as biomass, geothermal energy into power and it has the advantages of low maintenance requirements, high reliability and satisfying thermal performance. Besides that, it also shows great potential in the application of ICE waste heat recovery.
There exist large distinctions between cooling water heat and exhaust heat in terms of grade and quantity which makes it difficult to achieve high utilization efficiency for both of them. In most cases, focuses have been mainly given on exhaust waste heat recovery and that of cooling water is either not considered, or not completely utilized. On the other hand, the dynamic characteristics of actual engine operation result in an unsteady thermal boundary condition for WHR which is one of the greatest challenges for WHR-ORC system [2]. While compared with exhaust waste heat, cooling water is relatively steady as a heat source and its temperature rarely exceeds $95^{\circ} \mathrm{C}$ to prevent the phase change . Besides that, exhaust gases contain many acid compounds and the pressure drop does not allow for dramatic changes while flows into ORC system as it may penalize the normal operation of ICE which is an obstacle need to be overcome. In this work adequate solutions and suitable working fluids are studied to exploit cooling water heat, thus improving fuel utilization efficiency of ICE.

\section{SYSTEMS DESCRIPTION}

Basically, the outlet temperature of cooling water is rarely more than $95^{\circ} \mathrm{C}$, in this paper it's set to be $90^{\circ} \mathrm{C}$, while its mass flow rate is equal to $1 \mathrm{~kg} / \mathrm{s}$ to depict the general characteristic of any engine. Apart from that, the minimum return temperature should 
be no lower than $70^{\circ} \mathrm{C}$, as a too small value penalizes ICE fuel economy.

\subsection{Thermodynamic analysis of investigated ORC configurations}

The considered ORC layout configuration is shown in Fig.1. Conventional ORC consists of four main components, i.e. pump, evaporator, turbine and condenser. The low-pressure working fluid is pumped to high pressure. It then flows into the evaporator and heated by jacket water, after that, it converts heat into mechanical power by expansion in the turbine. Eventually it discharges unused residual heat through condensation.

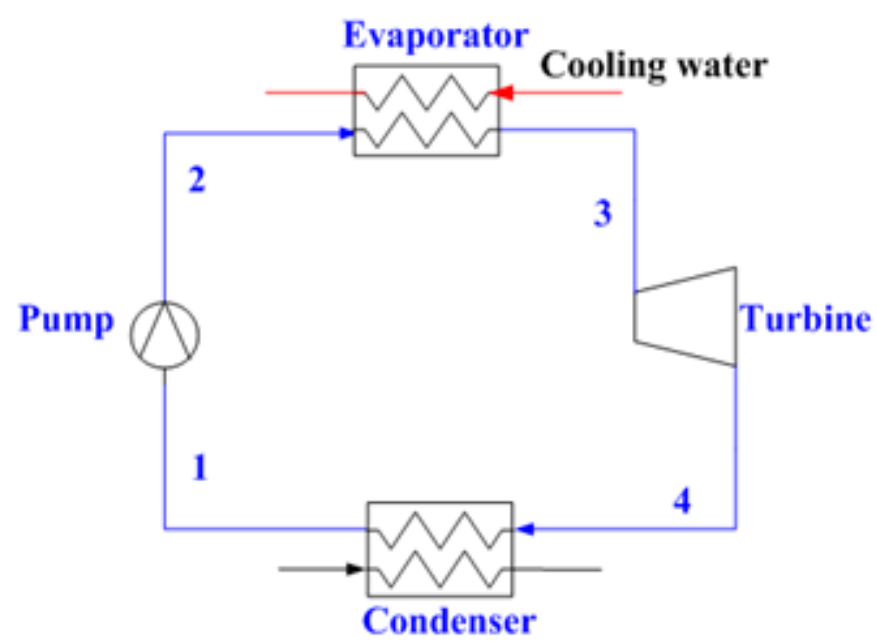

Fig.1 ORC layout configuration

In order to simplify the model, some assumptions are made. Specifically, all processes operate in steady states. The pressure drop and heat rejection of the pipes are negligible.

Based on the first thermodynamic law, energy investigation is conducted, and the corresponding mathematical model for each ORC configuration is collected in Table 1. While Table 2 gives the operating parameters for ORC.

According to the Montreal Protocol [9], many refrigerants were or will be phased out in the near future due to the environmental impact, especially the depletion of the ozone layer, apart from that, global warming also attracts increasingly attention. Calm and Hourahan pointed out that hydrocarbons are promising candidates of the next generation of refrigerants for the characteristics of zero/low ozone depletion potential (ODP), low global warming potential (GWP) and high efficiency[10,11]. The main concern against the application of hydrocarbons is flammability which can be avoided through adequate safety precautions, and it's noticeable that currently there are no working fluids available that completely meet all requirements including energy efficiency, safety, environment friendliness etc. In our work, butane, isobutane, pentane, isopentane are included for consideration. Their thermodynamic characteristics are collected in Table 3.

Table 1 Mathematical equations for each thermal process

\begin{tabular}{|c|c|}
\hline Component & Energy analysis \\
\hline Pump & $\eta_{p}=\frac{h_{2, s e}-h_{1}}{h_{2}-h_{1}}$ \\
\hline Evaporator & $Q_{n p}=m_{f}\left(h_{3}-h_{2}\right)=m_{c} \cdot c_{p} \cdot\left(T_{c, n}-T_{c, a e}\right)$ \\
\hline Turbine & $\eta_{t}=\frac{h_{3}-h_{4}}{h_{3}-h_{4, i t}}$ \\
\hline Condenser & $Q_{\text {ond }}=m_{f}\left(h_{4}-h_{1}\right)$ \\
\hline System & $W_{s t}=W_{t}+W_{p} \quad \quad \eta_{t s}=\frac{W_{s t}}{Q_{n p}}$ \\
\hline
\end{tabular}

Table 2 Operating parameters for ORC system

\begin{tabular}{|l|r|}
\hline Parameters & Values \\
\hline Pump isentropic efficiency, $\eta_{p}$ & 0.8 \\
\hline Turbine isentropic efficiency, $\eta_{t}$ & 0.75 \\
\hline ORC condensation temperature(K) & 303 \\
\hline Pinch temperature difference in the evaporator(K) & 5 \\
\hline Pinch temperature difference in the condenser(K) & 3 \\
\hline
\end{tabular}

Table 3 Working fluids' thermodynamic characteristics

\begin{tabular}{|l|l|l|}
\hline fluid & $T_{\text {crit }}(\mathrm{K})$ & $P_{\text {crit }}(\mathrm{MPa})$ \\
\hline butane & 425.13 & 3.796 \\
\hline isobutane & 407.81 & 3.629 \\
\hline pentane & 469.7 & 3.37 \\
\hline isopentane & 460.35 & 3.378 \\
\hline
\end{tabular}

\section{RESULTS AND DISCUSSION}

Fig.2 gives system net output power for these four working fluids. In particular, all cases share the identical tendency. System output power increases dramatically at first, and then experiences a fall. This is because when the evaporating pressure is relatively low, the corresponding evaporating temperature is small as well, and there exists relatively large temperature difference between the engine cooling water and working fluid, which means the heat transfer process happened in the evaporator cannot meet the pinch point temperature limitation and engine cooling water leaves the evaporator with a constant temperature (i.e the minimal allowable outlet temperature $70^{\circ} \mathrm{C}$ ). Hence large amount cooling water heat is released to ORC working fluid. Fig 3 shows the variation of system thermal efficiency with evaporating pressure, with the increase of evaporating, system thermal efficiency shows a consistent growth trend for all 
fluids which is in accordance with former researches [4-6]. While the evaporating pressure rises to a certain extent, as a result of pinch point temperature limitation in the evaporator, its increase leads to an higher engine cooling water outlet temperature, even though system thermal efficiency keeps growing, the waste heat released into the ORC system declines more significantly. Hence system output power sees a fall as well. Butane obtains the maximum output power of around $6.33 \mathrm{~kW}$ when the evaporating pressure is $0.8 \mathrm{MPa}$, followed by isobutane, isopentane and pentane.

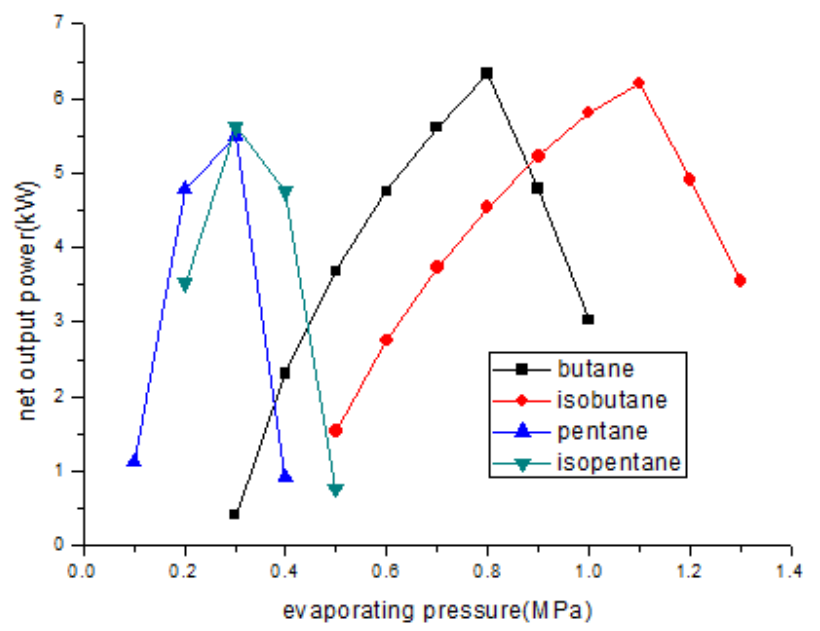

Fig.2 system net output power $(\mathrm{kW})$

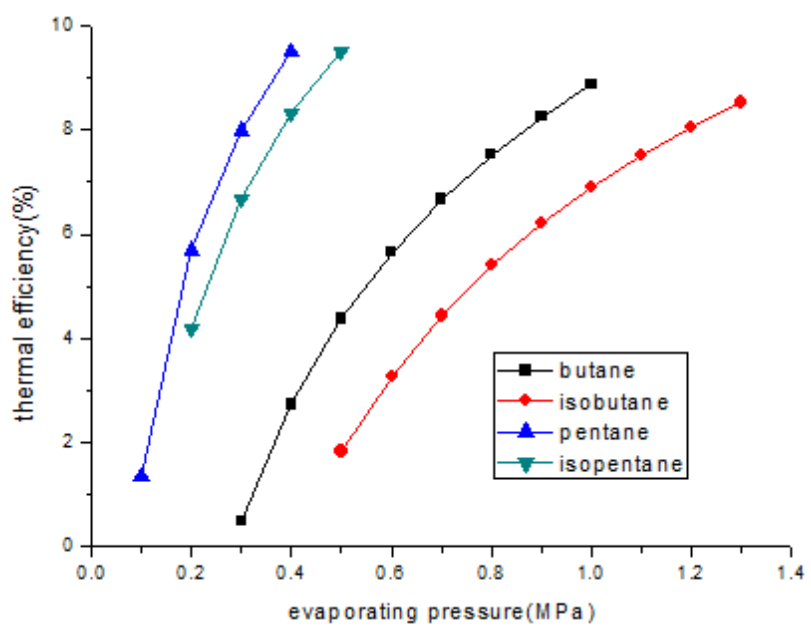

Fig.3 system thermal efficiency(\%)

Regarding the mass flow rate of working fluids, as can be observed from Fig.4, the mass flow rate decreases continuously with the rise of evaporating pressure. Specifically, it declines gently at first when the evaporating pressure is relatively small and engine cooling water leaves the evaporator with a constant temperature. Afterwards, it drops drastically as the outlet temperature of engine cooling water decreases obviously and less waste heat is utilized to heat working fluid.

Besides system energy recovery capacity, system size is another important aspect worth considering. This can be evaluated by introducing an index, i.e. volumetric flow rate at turbine inlet (see Fig.5). It's interesting that as evaporating pressure rises, the volumetric flow rate for pentane decreases dramatically, varying from 0.077 to around 0.001 $\mathrm{m}^{3} / \mathrm{s}$, implying an obvious turbine size. The decrease trends for both butane and isobutane are quite moderate.

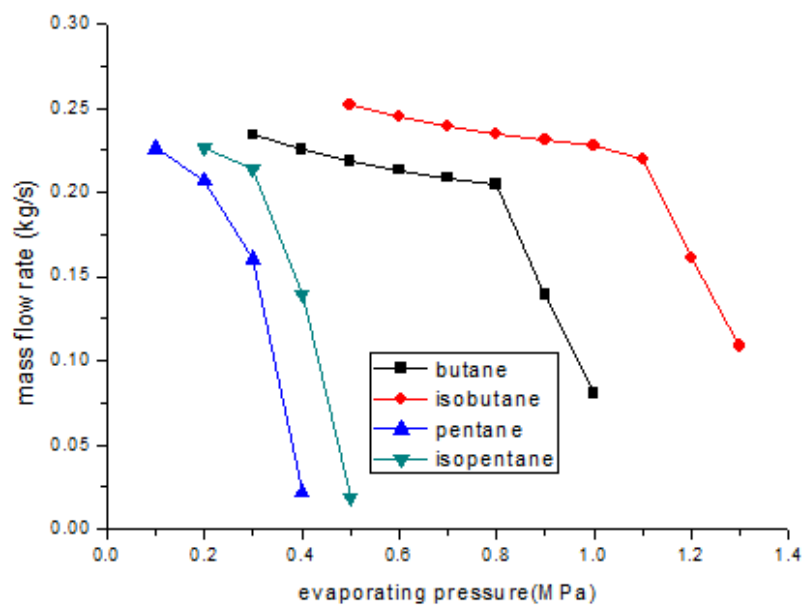

Fig.4 working fluid mass flow rate $(\mathrm{kg} / \mathrm{s})$

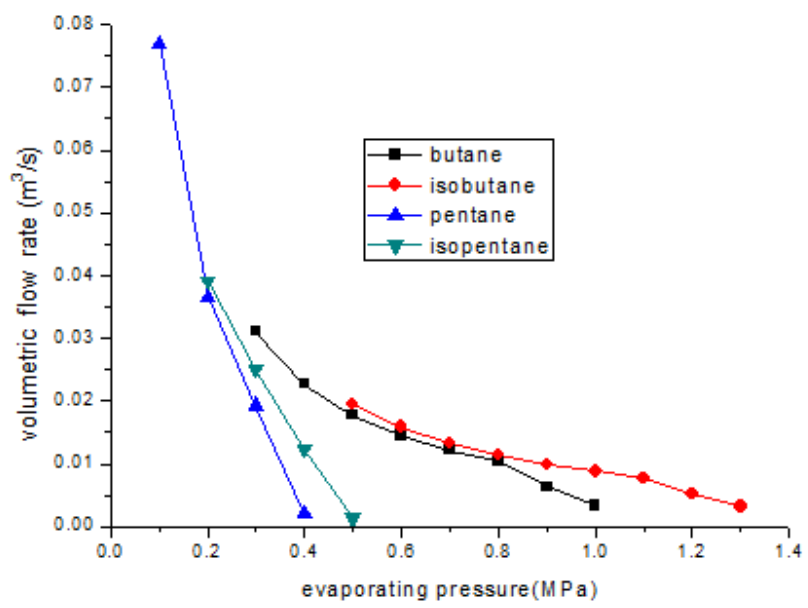

Fig. 5 volumetric flow rate at turbine inlet $\left(\mathrm{m}^{3} / \mathrm{s}\right)$

\section{CONCLUSIONS}

Aimed to recover engine cooling water waste heat complete, we conducted parametric optimization and working fluid selection for ORC system. Four environmentally friendly hydrocarbons, namely butane, isobutane, pentane and isopentane are selected for investigation. Main conclusions are drawn as follows:

(1) Due to the pinch point limitation in the evaporator, there exists a maximum output power for different working fluids, and butane reaches the peak of around $6.33 \mathrm{~kW}$ when the evaporating pressure is $0.8 \mathrm{MPa}$, followed by isobutane, isopentane and pentane.

(2) For the whole operating regions, pentane large thermal efficiency, and its volumetric flow rate decreases dramatically. System mass flow rate and 
volumetric flow rate see a fall with the increase of evaporating pressure.

\section{REFERENCES}

[1] Fu J, Liu J, Feng R, Wang YY, Wang Y, Energy and exergy analysis on gasoline engine based on mapping characteristics experiment. Applied Energy 2013; 102:622-630.

[2] Xie H, Yang C. Dynamic behavior of Rankine cycle system for waste heat recovery of heavy duty diesel engines under driving cycle. Applied Energy 2013; 112: $130-141$.

[3] Wang T, Zhang Y, Zhang J, et al. Comparisons of system benefits and thermoeconomics for exhaust energy recovery applied on a heavy-duty diesel engine and a light-duty vehicle gasoline engine. Energy Conversion and Management 2014; 84:97-107.

[4] Wei D, Lu X, Lu Z, et al. Performance analysis and optimization of organic Rankine cycle (ORC) for waste heat recovery. Energy Conversion and Management 2007; 48:1113-9.

[5] Kaska O. Energy and exergy analysis of an organic Rankine for power generation from waste heat recovery in steel industry. Energy Conversion and Management 2014; 77:108-17.

[6] Tian H, Shu G, Wei H, et al. Fluids and parameters optimization for the organic Rankine cycles (ORCs) used in exhaust heat recovery of Internal Combustion Engine (ICE). Energy 2012; 47:125-36.

[7] Wang EH, Zhang HG, Fan BY, et al. Study of working fluid selection of organic Rankine cycle (ORC) for engine waste heat recovery. Energy 2011; 36: 3406-18.

[8] Guo T, Wang HX, Zhang SJ. Selection of working fluids for a novel low temperature geothermally-powered ORC based cogeneration system. Energy Conversion and Management 2011; 52(6):2384-91.
[9] Government A. Montreal protocol on substances that deplete the ozone layer.Canberra 2013.

[10] Calm JM. The next generation of refrigerantsehistorical review, considerations, and outlook. Int J Refriger 2008; 31(7):1123-33.

[11] Calm JM, Hourahan GC. Physical, safety, and environmental data for current and alternative regrigerants. In: The 23rd international congress of refrigeration Prague. Czech Republic: International Institute of Refrigeration (IIR/IIF); 2011.

\section{APPENDIX}

\begin{tabular}{|c|c|}
\hline \multicolumn{2}{|c|}{ Nomenclature } \\
\hline $\mathrm{cp}$ & specific heat $(\mathrm{kJ} / \mathrm{kg} \bullet \mathrm{K})$ \\
\hline $\mathrm{h}$ & specific enthalpy $(\mathrm{kJ} / \mathrm{kg})$ \\
\hline $\mathrm{m}$ & mass flow rate $(\mathrm{kg} / \mathrm{s})$ \\
\hline Q & heat $(\mathrm{kW})$ \\
\hline $\mathrm{W}$ & power $(\mathrm{kW})$ \\
\hline \multicolumn{2}{|c|}{ Greek letters } \\
\hline$\eta$ th & thermal efficiency \\
\hline \multicolumn{2}{|c|}{ Subscripts } \\
\hline $\mathrm{c}$ & engine cooling water \\
\hline cond & condensation \\
\hline evp & evaporation \\
\hline$f$ & working fluid \\
\hline net & net output \\
\hline $\mathrm{P}$ & pump \\
\hline $\mathrm{t}$ & turbine \\
\hline
\end{tabular}

